\title{
Growth hormone protects colorectal cancer cells from radiation by improving the ability of DNA damage repair
}

\author{
XIAO-YU WU ${ }^{1 *}$, CHE CHEN $^{1 *}$, XUE-QUAN YAO ${ }^{1}$, QIN-HONG CAO ${ }^{1}$, \\ ZHE XU ${ }^{1}$, WEI-SU LI ${ }^{1}$, FU-KUN LIU ${ }^{1}$ and GANG LI ${ }^{2}$ \\ ${ }^{1}$ Department of Gastrointestinal Tumor Surgery, Affiliated Hospital of Nanjing University of Traditional Chinese Medicine, \\ Nanjing, Jiangsu 210029; ${ }^{2}$ Department of General Surgery, Jiangsu Cancer Hospital, \\ Affiliated Cancer Hospital of Nanjing Medical University, Nanjing, Jiangsu 210009, P.R. China
}

Received October 25, 2013; Accepted March 3, 2014

DOI: $10.3892 / \mathrm{mmr} .2014 .2185$

\begin{abstract}
The present study aimed to examine the effects of recombinant human growth hormone (rhGH) on the sensitivity of a colorectal cancer cell line to radiotherapy, and to investigate its association with DNA damage and repair. Flow cytometry and immunofluorescence were employed to detect growth hormone receptor (GHR) expression in nine human colorectal cancer cell lines. A colony forming assay was performed to measure the colorectal cancer cell proliferation post-radiotherapy, as an indicator of radiotherapy sensitivity. The comet assay results were interpreted as an indicator of radiotherapy-induced DNA damage, and growth arrest and DNA damage 45 (GADD45) and apurinic/apyrimidinic endonuclease (APEN) protein expression were quantified with western blot analysis from the same cell lines. The results demonstrated that the colony-forming efficiency (CFE) was significantly increased in HCT-8 cells subject to radiotherapy and $\mathrm{rhGH}$ pretreatment compared with the cells treated with radiotherapy alone, in a dose-dependent manner (0-100 mg/l). This effect was enhanced under high doses of radiation (8 Gy; $52.1 \pm 2.9$ vs. $21.0 \pm 2.7 ; \mathrm{P}<0.001)$ and was ameliorated with GHR neutralizing antibody exposure. By contrast, rhGH pre-incubation did not change the colony formation rate in GHR(-) LOVO cells. rhGH intervention reduced the early HCT-8 cell DNA damage $(21.53 \pm 2.88$ vs. $36.56 \pm 3.93$; $\mathrm{P}=0.003)$ as well as the following plateau phase, compared with cells treated with radiotherapy alone $(5.5 \pm 0.42$ vs. $9.07 \pm 0.84$; $\mathrm{P}=0.012)$. rhGH upregulated GADD45 and APEN protein expression, which is associated with cellular stress responses and DNA damage repair $(\mathrm{P}=0.007)$. The results suggest that
\end{abstract}

Correspondence to: Dr Gang Li, Department of General Surgery, Jiangsu Cancer Hospital, Affiliated Cancer Hospital of Nanjing Medical University, 42 Baiziting, Nanjing, Jiangsu 210009, P.R. China

E-mail: searain@sohu.com

*Contributed equally

Key words: growth hormone, colorectal cancer, radiotherapy, DNA damage repair
rhGH is able to protect colorectal cancer cells from radiation through the interaction with GHR, which is associated with the promotion of DNA damage repair activity.

\section{Introduction}

In our previous pre-clinical study, it was identified that colorectal cancer patients, who have a high expression level of growth hormone receptor (GHR), are radioresistant (1), indicating that GH/GHR signaling may be associated with cellular stress responses to radiotherapy. Using Chinese hamster ovary cells (CHO-4) in vitro, Madrid et al (2) found that growth hormone (GH) was able to reduce the cell damage induced by radiotherapy through its association with GHR on the cell surface. Isla et al $(3,4)$ also identified that $\mathrm{GH}$ is involved in protection against the noxious effects of radiotherapy in the spinal cords of rats and in cell cultures of the central nervous systems. A recent study demonstrated that autocrine human growth hormone is able to protect mammary carcinoma cells from the induction of DNA double-strand breaks following chemotherapy (5).

Based on these preliminary data, the current study aimed to investigate whether recombinant human growth hormone $(\mathrm{rhGH})$ also has a role in the protection of colorectal cancer cells post-radiotherapy, as it does in the protection of normal healthy cells.

\section{Materials and methods}

Detection of GHR expression on the colorectal cancer cell surface by flow cytometry. Nine colorectal cancer cell lines were provided by Shanghai Institute of Biochemistry and Cell Biology (Shanghai, China), including LOVO, HCT-8, SW480, Ls-174-T, HT-29, CL187/CCL187, COLO320/DM, COLO205 and HCE8693. A mouse monoclonal antibody against GHR was provided by Sigma-Aldrich (St. Louis, MO, USA). phycoerythrin (PE)-labeled goat anti-mouse IgG1 secondary antibody was provided by Caltag Laboratories (Carlsbad, CA, USA). PE-labeled mouse IgG1 was provided by R\&D Systems (Emeryville, CA, USA) as the control. All of the cell lines were cultured in RPMI-1640 medium at $37^{\circ} \mathrm{C}$ in $5 \%$ $\mathrm{CO}_{2}$. Flow cytometry was conducted according to standard procedures. 
Experimental grouping. GHR positive HCT-8 cells and GHR negative LOVO cells were selected from nine colorectal cancer cell lines. Recombinant human growth hormone (rhGH, Saizen) was provided by Serono (no. 1260411D04). The GHR antagonist (GHRA) is the goat anti-human GHR antibody, which actively neutralizes human GH and was provided by R\&D Systems. The median neutralization concentration (ND50) of GHRA was $0.125-0.5 \mu \mathrm{g} / \mathrm{ml}$.

Serum starvation was conducted for the HCT- 8 cells $(16 \mathrm{~h})$ and LOVO cells $(8 \mathrm{~h})$ prior to the experiments. The cells were radiated following $6 \mathrm{~h}$ treatment of rhGH and GHRA pretreatment was conducted $1 \mathrm{~h}$ prior to rhGH treatment. Radiation was performed by an Elekta1070 linear accelerator (Varian Medical Systems, Palo Alto, CA, USA) under room temperature with a dose series of 2, 4 and $8 \mathrm{~Gy}$, respectively and a dose rate of $100 \mathrm{cGy} / \mathrm{min}$. Following radiation, the cells were collected at various time points according to different samples and each group had five replicates. The experimental grouping is summarized in Table I.

Colony forming assay. A colony forming assay is the gold standard for determining cell proliferation (6). Following treatment and radiation, the cells from the culture flask were digested into a single cell suspension. The cells of the suspension were inoculated in a petri dish with $10 \mathrm{ml}$ pre-heated medium at a density of 500 cells/dish. The cells were cultured for 2-3 weeks under the conditions of $5 \% \mathrm{CO}_{2}, 37^{\circ} \mathrm{C}$ and saturated humidity. Following detection of the colonies by the naked eye, the cells were stained and fixed by Giemsa stain and dried in air for 10-30 min. The number of colonies containing $>50$ cells were counted. The survival fraction (SF) was calculated using the following formula: $\mathrm{SF}=$ number of colonies $/$ (number of inoculated colonies x PE/100), where PE is the colony formation rate under certain conditions. The control group without treatment was used as a standard.

Detection of DNA damage by comet assay. A comet assay is also known as Single Cell Gel Electrophoresis (SCGE) and is considered the standard method for determining DNA damage (7). A number of the steps of SCGE were modified according to our laboratory conditions (Nanjing University of Traditional Chinese Medicine, Nanjing, Jiangsu, China) (8), including cell separation and treatment, constructing slides, cell lysis, DNA denaturation, signal cell electrophoresis, neutralization, staining. The images were analyzed by Comet Assay Software Project (CASP) Perceptive Instruments (London, UK). A total of 50 cells were selected randomly and detected at olive tail moment (OTM) from each experimental group. OTM is a common index to determine the DNA damage level in a comet assay (9) and is defined as the product of the tail length and the fraction of total DNA in the tail. The DNA repair process most commonly requires 4-6 h following radiation and therefore the DNA damage was detected at different time points of $0,15,30,60,60,120$ and $240 \mathrm{~min}$ following radiation. The concentration of rhGH and GHRA were $100 \mathrm{ng} / \mathrm{ml}$ and $0.2 \mu \mathrm{g} / \mathrm{ml}$, respectively and the radiation dose was $8 \mathrm{~Gy}$.

Detection of the gene expression level of growth arrest and DNA damage 45 (GADD45) and apuriniclapyrimidinic endonuclease (APEN) following rhGH treatment by western blot
Table I. Experimental grouping for examining the effects of rhGH on human colorectal cancer cell radiosensitivity.

\begin{tabular}{lcc}
\hline Group & HCT-8 (GHR+) & LOVO (GHR-) \\
\hline Radiation & 1 & A \\
rhGH $^{\mathrm{a}}+$ radiation & 2 & B \\
GHRA $^{\mathrm{b}}+$ rhGH + radiation & 3 & C
\end{tabular}

aThe concentration of rhGH is 5,25 and $100 \mathrm{ng} / \mathrm{ml}$, respectively; b the concentration of GHRA is $0.2 \mu \mathrm{g} / \mathrm{ml}$. rhGH, recombinant human growth hormone; GHR, growth hormone receptor; GHRA, growth hormone reaceptor antagonist.

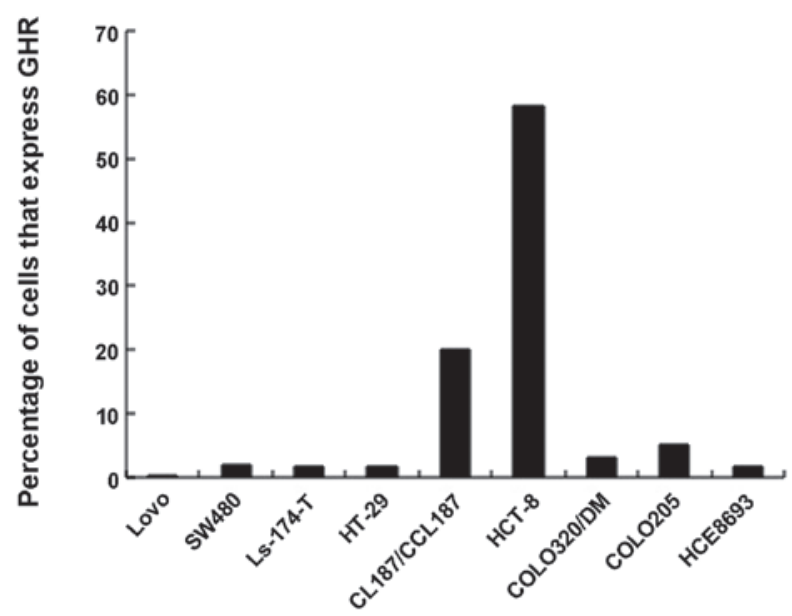

Figure 1. Percentage of colorectal cancer cells that expressed GHR on the cell surface. GHR, growth hormone receptor.

analysis. GADD45 and APEN protein levels were detected in GHR(+) HCT-8 cells 1, 3 and 6 h following rhGH treatment $(100 \mathrm{ng} / \mathrm{ml})$. Each experiment was performed as three replicates. GADD45 and APEN polyclonal antibodies were purchased from Santa Cruz Biotechnology Inc. (Santa Cruz, CA, USA). Western blot analysis was performed according to standard procedures.

Statistical analysis. The results are presented as the mean \pm standard deviation. The differences between the groups were analyzed by single element variance analysis and $\mathrm{P}<0.05$ was considered to indicate a statistically significant difference. Data were analyzed by statistics software SPSS 11.0 (SPSS, Inc., Chicago, IL, USA).

\section{Results}

Expression level of GHR on the surface of colorectal cancer cells. The expression level of GHR in human colorectal cancer cells was investigated using flow cytometry. It was identified that the cell lines, including LOVO, SW480, Ls-174-T, HT-29, COLO320/DM, COLO205 and HCE8693 did not express or expressed very low level of GHR. CL187/CCL187 expressed a certain level of GHR (19.99\% of cells expressed GHR) and HCT-8 expressed a high level of GHR (58.23\% of cells expressed GHR; Fig. 1). For the further experiments, HCT-8 
Table II. Effects of rhGH on HCT-8 DNA repair following radiation.

Time after radiation $(\min )$

\begin{tabular}{|c|c|c|c|c|c|c|}
\hline \multirow[b]{2}{*}{ Group } & \\
\hline & 0 & 15 & 30 & 60 & 120 & 240 \\
\hline Control & $0.78 \pm 0.08$ & $1.47 \pm 0.15$ & $0.95 \pm 0.10$ & $1.24 \pm 0.07$ & $1.09 \pm 0.09$ & $0.75 \pm 0.10$ \\
\hline Radiation $^{c}$ & $36.56 \pm 3.93$ & $22.4 \pm 1.91$ & $14.68 \pm 1.30$ & $10.69 \pm 0.99$ & $9.07 \pm 0.84$ & $9.49 \pm 0.94$ \\
\hline $\mathrm{rhGH}^{\mathrm{a}}+$ radiation & $21.53 \pm 2.88^{* * *}$ & $13.78 \pm 1.25^{*}$ & $9.35 \pm 1.05^{*}$ & $6.05 \pm 0.79^{*}$ & $5.5 \pm 0.42^{*}$ & $5.65 \pm 0.62^{*}$ \\
\hline $\mathrm{GHRA}^{\mathrm{b}}+\mathrm{rhGH}+$ radiation & $39.45 \pm 3.59$ & $19.5 \pm 2.21$ & $15.9 \pm 1.76$ & $9.97 \pm 1.25$ & $8.61 \pm 0.64$ & $8.85 \pm 0.91$ \\
\hline
\end{tabular}

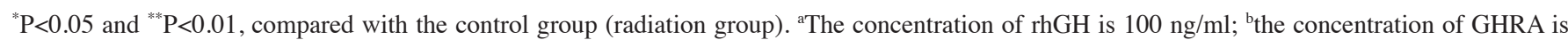

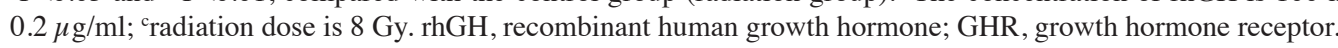

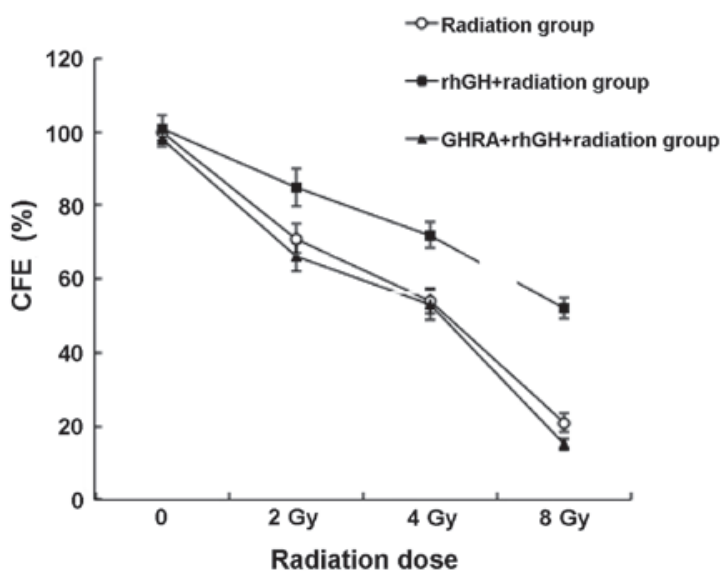

Figure 2. Effects of rhGH treatment on the CFE of HCT-8 cells post-radiotherapy. CFE was presented as a percentage of the control cells. rhGH, recombinant human growth hormone; GHRA, growth hormone receptor antagonist; CFE, colony formation efficiency.

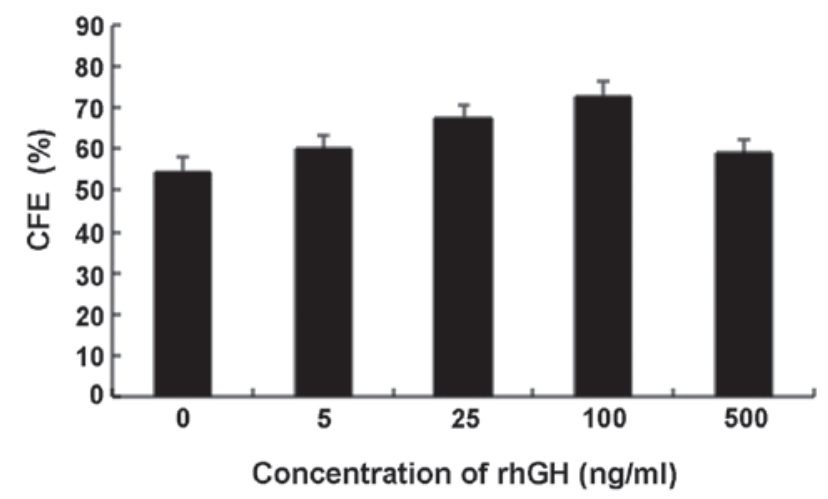

Figure 3. Effects of different rhGH concentrations on the CFE of HCT-8 cells post-radiotherapy. rhGH, recombinant human growth hormone; CFE, colony formation efficiency.

cells were selected as the GHR(+) cells and LOVO as the GHR(-) cells, which acted as a control.

Effects of rhGH treatment on the colony forming efficiency (CFE) of colorectal cancer cells following radiation. As demonstrated in Fig. 2, when treated with $100 \mathrm{ng} / \mathrm{ml} \mathrm{rhGH}$, post-radiotherapy, HCT-8 cells had a significantly higher CFE than the control group, particularly under a radiation dose of 8 Gy $(52.1 \pm 2.9$ vs. $21.0 \pm 2.7 ; \mathrm{P}<0.001)$. To investigate whether

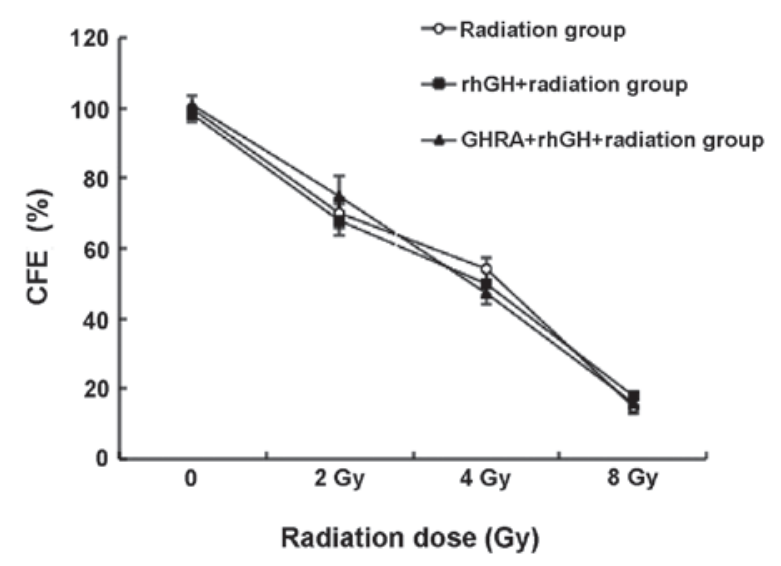

Figure 4. Effects of rhGH on the CFE of GHR(-) LOVO cells following radiation. rhGH, recombinant human growth hormone; GHRA, growth hormone receptor anatagonist; CFE, colony formation efficiency.

this effect was dependent on the concentration of rhGH, the $\mathrm{CFE}$ of cells treated with various concentrations of rhGH was examined. Notably, it was identified that the CFE was only dependent on the concentration of rhGH within a certain range $(0-100 \mathrm{ng} / \mathrm{ml}$; Fig. 3). Above a concentration of $100 \mathrm{ng} / \mathrm{ml}$, rhGH began to reduce the CFE of the cells.

To rule out the possibility that rhGH alone, rather than the association of rhGH with GHR, increased the CFE, an antagonist was utilized to prevent GHR from binding to rhGH (Fig. 2). With the treatment of GHRA, it was identified that the CFE decreased to a similar level of the control even in the presence of rhGH, suggesting that the protective function of rhGH was eliminated by pretreating GHR with GHRA.

To further confirm that rhGH functions through GHR, the CFE of GHR(-) LOVO cells post-radiotherapy was detected. Consistent with the previous results, rhGH treatment did not significantly increase the CFE of GHR(-) LOVO cells following radiation ( $\mathrm{P}>0.05$; Fig. 4). Following the GHRA treatment, no significant difference was found between the GHRA and GHR treatment, and the control group $(\mathrm{P}>0.05)$. Similarly, changing the concentration of rhGH did not affect the level of CFE (data not presented), indicating rhGH only functions in the presence of GHR.

Effects of rhGH treatment on DNA repair in post-radiotherapy colorectal cancer cells. To further investigate the mechanism 


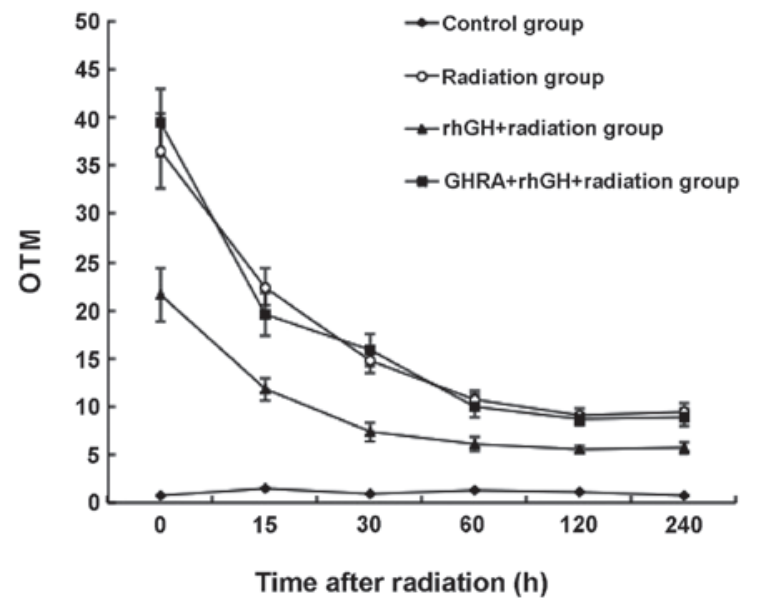

Figure 5. Effects of rhGH on HCT-8 DNA repair following radiation. rhGH, recombinant human growth hormone; GHR, growth hormone receptor; OTM, olive tail moment.

$\mathbf{A}$

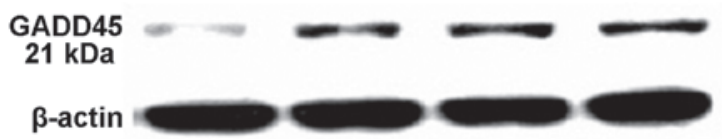

B

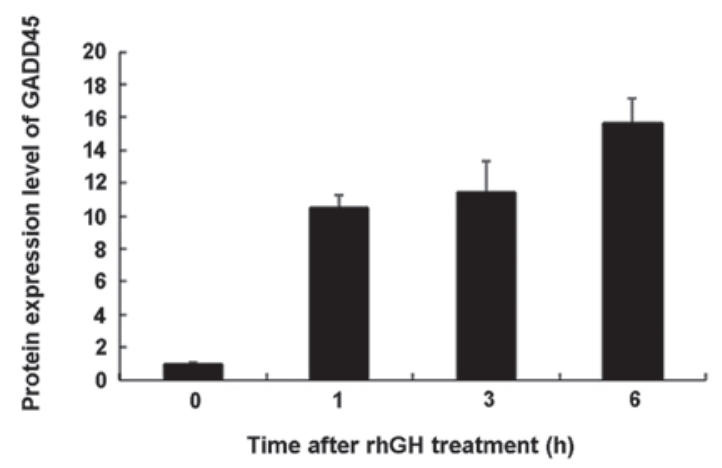

Figure 6. GADD45 protein expression level is upregulated by rhGH. (A) Protein levels of GADD45 demonstrated by western blot analysis. (B) The quantification of GADD45 protein level. rhGH, recombinant human growth hormone; GADD45, growth arrest and DNA damage 45.

of the protective function of rhGH, a comet assay was used to determine the extent of DNA damage in the cells. As demonstrated in the radiation alone group in Table II and Fig. 5, the DNA damage level of HCT-8 cells was highest immediately after radiation and gradually reached the plateau phase within 120 min through continuous DNA repair. Treatment of rhGH resulted in a significantly lower level of DNA damage in the HCT- 8 cells $(21.53 \pm 2.88$ vs. $36.56 \pm 3.93$ in the control group; $\mathrm{P}=0.003$ ) and the level in plateau phase was also significantly lower than those cells without rhGH treatment $(5.5 \pm 0.42$ vs. $9.07 \pm 0.84$ in the control group, $\mathrm{P}=0.012$ ). Following GHRA pretreatment, the DNA damage level returned to a similar level compared with the control group.

rhGH upregulates the protein expression level of GADD45 and APEN in colorectal cancer cells. Since the mechanisms underlying the protective effects of rhGH remain obscure, the correlation between rhGH and two proteins that are associated
A

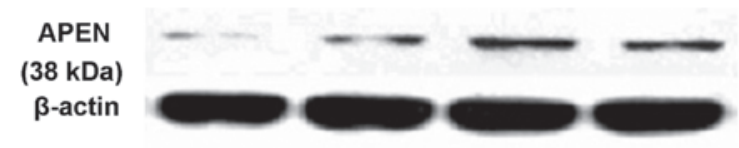

B

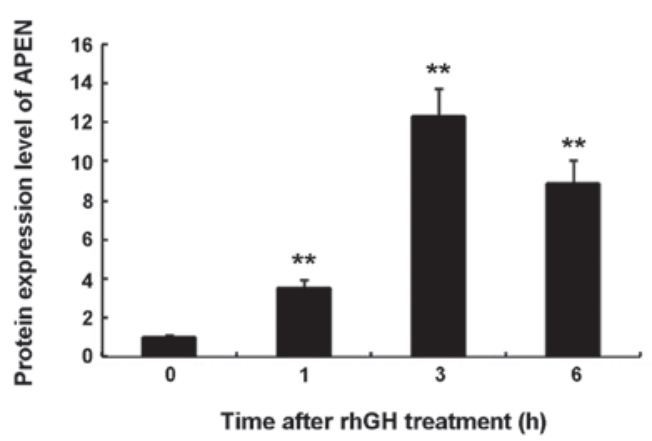

Figure 7. (A) APEN protein expression level is upregulated by rhGH. (B) Error bars indicate the quantification of the APEN protein level. rhGH, recombinant human growth hormone; APEN, apurinic/apyrimidinic endonuclease,

with DNA repair was examined. As demonstrated in Figs. 6 and 7, the protein expression levels of GADD45 and APEN were significantly upregulated following $1 \mathrm{~h}$ of treatment with rhGH $(\mathrm{P}<0.001)$ and the high expression level was maintained for as long as $6 \mathrm{~h}(\mathrm{P}<0.001)$.

\section{Discussion}

In the present study, it was demonstrated that exogenous growth hormone significantly increased the CFE of HCT-8 following radiation and the level of CFE was dependent on the concentration of rhGH within a specific concentration range $(0-100 \mathrm{ng} / \mathrm{ml})$. However, the same results were not observed in the GHR(-) LOVO cells. Furthermore, when GHR was blocked by pretreatment of GHRA, rhGH failed to increase the CFE level of HCT-8 cells. These results suggest that GH has an important role in protecting GHR(+) colorectal cancer cells from radiation and that this protective mechanism is mediated through its interaction with GHR on the cell surface. Of note, when applying rhGH at a markedly high concentration, the protective effect was no longer evident, which may be attributed to the inhibition of interaction between GH and GHR (10). Under normal conditions, one molecule of rhGH requires two GHRs in order to form a dimer and transduce a signal into cells. When the concentration of rhGH is particularly high, one rhGH molecule will only bind to one GHR and therefore does not form a functional dimer and thus fails to transduce a biological signal into cells.

DNA is the major target of the damaging effects of radiation. The DNA damage caused by radiation and the ability of DNA to repair in cells affects the cellular activity and overall viability. Following exposure to radiation, cellular DNA repair occurs within 4-6 h, during which the cells with mild DNA damage enter mitosis while cells with severe damage are induced to enter apoptosis (11). In the present study, a comet assay was used to detect the DNA damage of GHR(+) HCT-8 cells at various time points within $4 \mathrm{~h}$ following radiation. It was identified that post-radiotherapy cells treated with rhGH had lower levels of DNA damage compared with the control group and the DNA damage level of rhGH-treated cells was 
also low at plateau phase. However, the protective effect was inhibited by blocking the cells with neutralizing antibody GHRA. This suggests that the association of GH and GHR improves the DNA repair ability in HCT-8 colorectal cancer cells, which may increase the CFE following radiation.

Previously, a study of the rat liver demonstrated that GH induces the expression of two genes implicated in the control of DNA damage and in cellular stress responses (12), namely, GADD45 and APEN. GADD45 is a nucleoprotein closely correlated with cellular DNA repair mechanisms. When DNA is damaged, GADD45 expression is increased and then prevents the cell from entering the $\mathrm{S}$ phase for DNA replication (13), while simultaneously activating nucleotide excision repair (NER). In addition, GADD45 is able to induce cell cycle arrest, predominantly via activating the G2 checkpoint (14). APEN is a member of a family of DNA repair enzymes and is ubiquitously expressed in various tissues. The main function of APEN is to excise apurinic/apyrimidinic (AP) bases on DNA via base excision repair (BER), thus maintaining genetic stability (15).

The interaction of GH with its receptor induces the activation of signaling cascades, including the janus kinase 2 (JAK2) tyrosine kinase (16), mitogen-activated protein kinase (MAPK) (17,18), insulin receptor substrate (IRS)-1 and IRS-2(19,20), phosphatidylinositol 3-kinase (21), Src homologous and collagen-like protein, growth factor receptor-bound protein 2 (22), protein kinase C and phospholipase A2 (23) and others. A number of these pathways, particularly those driven by JAK 2 and MAPK, activate transcription mediated by signal transducer and activator of transcription (STAT) 3 and STAT5 as well as SRF and c-fos (24-26), and possibly stimulate the expression of genes, such as GADD45 and APEN, which are involved in radiation-induced DNA repair. In the present study, it was also demonstrated that the association of GH and GHR significantly upregulated the expression level of GADD45 and APEN in HCT-8 colorectal cancer cells, which may enhance the ability of DNA to repair. These data may partly explain the molecular mechanism underlying the protective effects of GH.

Currently, whether rhGH may be used in patients with colorectal cancer remains controversial. A number of studies have combined GH and radiotherapy in order to improve the radiation resistance of cancer patients and enhance the efficacy. However, as indicated by the results of the present study, the protective effects of GH on colorectal cancer cells may, conversely, prevent the radiation-induced apoptosis of cancer cells. Therefore, the use of rhGH as a therapeutic approach may be limited in colorectal cancer. By contrast, the competition of GHRAs (such as Pegvisomant) (27) with GHR, blocks GH/GHR signaling and may increase the radiosensitivity of colorectal cancer cells.

In conclusion, the present study demonstrates that rhGH has an important role in the protection of colorectal cancer cells from radiation, and this protection may be associated with elevated DNA repair ability. Further studies are required to determine how GH/GHR signal transduction regulates feedback to radiotherapy-mediated cellular stress.

\section{References}

1. Wu X, Wan M, Li G, Xu Z, et al: Growth hormone receptor overexpression predicts response of rectal cancers to pre-operative radiotherapy. Eur J Cancer 42: 888-894, 2006.
2. Madrid O, Varea S, Sanchez-Perez I, et al: Growth hormone protects against radiotherapy-induced cell death. Eur J Endocrinol 147: 535-541, 2002.

3. Isla A, Budke M, García-Grande A, et al: Protective effects of the growth hormone $(\mathrm{GH})$ on the irradiated spinal cord in rats. Neurocirugia (Astur) 18: 89-94, 2007 (In Spanish).

4. Isla A, Budke M, Cacicedo L, et al: Protective effects of growth hormone in cell cultures of the central nervous system. Rev Neurol 34: 208-211, 2002 (In Spanish).

5. Bougen NM, Steiner M, Pertziger M, et al: Autocrine human GH promotes radioresistance in mammary and endometrial carcinoma cells. Endocr Relat Cancer 19: 625-644, 2012.

6. Dunne AL, Price ME, Mothersill C, et al: Relationship between clonogenic radiosensitivity, radiation-induced apoptosis and DNA damage/repair in human colon cancer cells. Br J Cancer 89: 2277-2283, 2003

7. Rojas E, Lopez MC and Valverde M: Single cell gel electrophoresis assay: methodology and applications. J Chromatogr B Biomed Sci Appl 722: 225-254, 1999.

8. Collins AR: The comet assay for DNA damage and repair: principles, applications, and limitations. Mol Biotechnol 26: 249-261, 2004.

9. Lee E, Oh E, Lee J, Sul D and Lee J: Use of the tail moment of the lymphocytes to evaluate DNA damage in human biomonitoring studies. Toxicol Sci 81: 121-132, 2004.

10. Fuh G, Cunningham BC, Fukunaga R, et al: Rational design of potent antagonists to the human growth hormone receptor. Science 256: 1677-1680, 1992.

11. Willers H, Dahm-Daphi J and Powell SN: Repair of radiation damage to DNA. Br J Cancer 90: 1297-1301, 2004.

12. Thompson BJ, Shang CA and Waters MJ: Identification of genes induced by growth hormone in rat liver using cDNA arrays. Endocrinology 141: 4321-4324, 2000.

13. Wang XW, Zhan Q, Coursen JD, et al: GADD45 induction of a G2/M cell cycle checkpoint. Proc Natl Acad Sci USA 96: 3706-3711, 1999

14. Liebermann DA and Hoffman B: Gadd45 in stress signaling. J Mol Signal 3: 15, 2008.

15. Fishel ML and Kelley MR: The DNA base excision repair protein Ape1/Ref-1 as a therapeutic and chemopreventive target. Mol Aspects Med 28: 375-395, 2007.

16. Argetsinger LS and Carter-Su C: Growth hormone signalling mechanisms: involvement of the tyrosine kinase JAK2. Horm Res 45 (Suppl 1): 22-24, 1996.

17. Love DW, Whatmore AJ, Clayton PE and Silva CM: Growth hormone stimulation of the mitogen-activated protein kinase pathway is cell type specific. Endocrinology 139: 1965-1971, 1998.

18. Möller C, Hansson A, Enberg B, et al: Growth hormone $(\mathrm{GH})$ induction of tyrosine phosphorylation and activation of mitogen-activated protein kinases in cells transfected with rat GH receptor cDNA. J Biol Chem 267: 23403-23408, 1992.

19. Argetsinger LS, Hsu GW, Myers MG Jr, et al: Growth hormone, interferon-gamma, and leukemia inhibitory factor promoted tyrosyl phosphorylation of insulin receptor substrate-1. J Biol Chem 270: 14685-14692, 1995

20. Argetsinger LS, Norstedt G, Billestrup N, et al: Growth hormone, interferon-gamma, and leukemia inhibitory factor utilize insulin receptor substrate-2 in intracellular signalling. J Biol Chem 271: 29415-29421, 1996.

21. Jeay S, Sonenshein GE, Kelly PA, et al: Growth hormone exerts antiapoptotic and proliferative effects through two different pathways involving nuclear factor-kappaB and phosphatidylinositol 3-kinase. Endocrinology 142: 147-156, 2001.

22. VanderKuur J, Allevato G, Billestrup N, et al: Growth hormone-promoted tyrosyl phosphorylation of SHC proteins and SHC association with Grb2. J Biol Chem 270: 7587-7593, 1995.

23. Argetsinger LS and Carter-Su C: Mechanism of signalling by growth hormone receptor. Physiol Rev 76: 1089-1107, 1996.

24. Wang YD and Wood WI: Amino acids of the human growth hormone receptor that are required for proliferation and Jak-STAT signalling. Mol Endocrinol 9: 303-311, 1995.

25. Pircher TJ, Flores-Morales A, Mui AL, et al: Mitogen-activated protein kinase inhibition decreases growth hormone stimulated transcription mediated by STAT5. Mol Cell Endocrinol 133: 169-176, 1997.

26. Ihle JN, Witthuhn BA, Quelle FW, et al: Signalling through the hematopoietic cytokine receptors. Ann Rev Immunol 13: 369-398, 1995.

27. Drake WM and Trainer PJ: Clinical use of pegvisomant for the treatment of acromegaly. Treat Endocrinol 2: 369-374, 2003. 